\title{
Avaliação do poder sensibilizante da reação de Montenegro
}

\author{
Evaluation of the ability of Montenegro skin test to induce specific immune response
}

\author{
Fábio Freire José ${ }^{\text {, Indiara Maria da Silva }}{ }^{1}$, Maria IIma Araújo, ${ }^{1,2}$, Roque P. Almeida ${ }^{2}$,
} Olívia Bacellar ${ }^{2}$ e Edgar M. Carvalho ${ }^{1,2}$

\begin{abstract}
Resumo O teste de Montenegro, utilizado no diagnóstico da leishmaniose tegumentar, tem sido recentemente considerado marcador de imunogenicidade após vacinação. Neste estudo, avaliou-se o poder sensibilizante deste teste e a produção de y-IFN in vitro, em indivíduos não expostos a Leishmania. Utilizaram-se para o teste antígenos de L. amazonensis produzido em nosso laboratório (grupo I) ou produzido pela FIOCRUZ-RJ (grupo II). No dia 30, 33\% dos indivíduos do grupo I e 42\% do grupo II converteram o teste para positivo, comparando-se com $67 \%$ do grupo I e $50 \%$ do grupo II no dia 90 . y-IFN foi detectado em $56 \%$ dos indivíduos do grupo I e 17\% do grupo II no dia $30(169 \pm 309$ e $11 \pm 36 \mathrm{pg} / \mathrm{ml})$ e em $67 \%$ do grupo I e $58 \%$ do grupo II no dia $360(69 \pm 107$ e $18 \pm 20 \mathrm{pg} / \mathrm{ml}$ ). Estes dados demonstram que o teste de Montenegro induz, além de hipersensibilidade tardia, a produção de y-IFN.
\end{abstract}

Palavras-chaves: Teste de Montenegro. Teste cutâneo de hipersensibilidade tardia. Diagnóstico de leishmaniose.

Abstract The Montenegro skin test, used to diagnose cutaneous leishmaniasis, is now being considered to detect immunogenicity after vaccination. In this study, we evaluated the ability of this test to induce immune response and IFN-g production in subjects not previously exposed to Leishmania. The Montenegro skin test was performed using antigens of $\mathrm{L}$. amazonensis produced by our laboratory (group I) or by FIOCRU-RJ (group II). At day 30, 33\% of the subjects from group I and $42 \%$ from group II were positive, compared to $67 \%$ from group I and $50 \%$ from group II at day 90 . IFN-y was detected in $56 \%$ of subjects from group I and $17 \%$ from group II at day $30(169 \pm 309$ and $11 \pm 36 \mathrm{pg} / \mathrm{ml})$ and in $67 \%$ from group I and $58 \%$ from group II by day 360 $(69 \pm 107$ and $18 \pm 20 \mathrm{pg} / \mathrm{ml})$. These data demonstrate that the Montenegro skin test induces not only a delayed hypersensitivity reaction, but also IFN-y production.

Key-words: Montenegro skin test. Delayed hypersensitivity skin test. Leishmaniasis diagnosis.

A intradermorreação idealizada por Montenegro ${ }^{27}$ representa o principal exame complementar para o diagnóstico da leishmaniose tegumentar americana (LTA) em áreas endêmicas, sendo, portanto, de relevante importância para países como o Brasil, que, juntamente, com o Afeganistão, Peru, Arábia Saudita e Síria detêm $90 \%$ dos casos de leishmaniose tegumentar em todo o mundo ${ }^{41}$. Essa reação de hipersensibilidade tardia possui sensibilidade variando entre 86 e $100 \%$ e especificidade de aproximadamente $100 \%{ }^{1011}$, o que a consagrou como uma das provas mais usadas na confirmação da doença ativa, no diagnóstico retrospectivo e em inquéritos epidemiológicos de LTA 1330313336 . Uma reação de Montenegro positiva em indivíduos de áreas endêmicas sem história de leishmaniose tegumentar americana e sem qualquer lesão suspeita, aponta para a possibilidade de formas abortivas ou infecções subclínicas ${ }^{11} 1220$.
A despeito da alta sensibilidade e especificidade do teste de Montenegro, existem relatos de reação cruzada, principalmente em indivíduos com doença de Chagas e indivíduos curados de leishmaniose visceral ${ }^{35}$. Resultados falsos negativos também têm sido descritos quando a infecção é precoce ${ }^{8}$, ou em casos em que a doença cutânea seja causada por Leishmania amazonensis ${ }^{37}$. Apesar do teste de Montenegro ser plenamente aceito, diferenças na preparação comercial podem afetar a antigenicidade e a eficácia na detecção de diversas formas de leishmaniose em diferentes áreas geográficas ${ }^{12}$.

A negatividade do teste intradérmico de Montenegro tem sido utilizado como critério de inclusão de indivíduos em estudos que visam caracterizar a resposta imune $e$ a eficácia da imunização após a utilização de vacinas contra leishmaniose ${ }^{4522232428}$. A demonstração de que o teste de Montenegro pode sensibilizar o hospedeiro

1. Escola Baiana de Medicina e Saúde Pública;2. Serviço de Imunologia, Hospital Universitário Professor Edgard Santos da Universidade Federal da Bahia, Salvador, Bahia, Brasil.

Endereço para correspondência: Dr. Edgar Marcelino de Carvalho. Serviço de Imunologia/Hospital Universitário Professor Edgard Santo/UFBA. R. João da Botas s/n, $5^{\circ}$ andar, 40140-160 Salvador, BA, Brasil.

Tel: 5573 237-7353; Fax: 5571 245-7110.

Recebido para publicação em 2/4/2001. 
e fazer com que, em uma segunda oportunidade, a reação torne-se positiva, compromete a utilização do mesmo como indicador de proteção após vacinação ${ }^{29}$. Estudo in vitro avaliando a resposta linfoproliferativa de indivíduos vacinados para leishmaniose e indivíduos que se submeteram ao teste de Montenegro, porém não receberam a vacina, não mostrando diferenças significativas entre os grupos ${ }^{26}$.

\section{MATERIAL E MÉTODOS}

Indivíduos estudados. Participaram do estudo 24 indivíduos sadios recrutados entre alunos do curso médico e profissionais da área de saúde, de ambos os sexos, com idades variando entre 18 e 30 anos, sem história prévia de leishmaniose, não residentes e não procedentes de áreas endêmicas da doença e que, durante o período do estudo, tivessem baixo risco de serem expostos à infecção por Leishmania. Os indivíduos negavam alergias, tinham sorologia negativa para HIV, e epidemiologia negativa para doença de Chagas. A participação no estudo teve caráter voluntário e os indivíduos assinaram um termo de consentimento. $\mathrm{O}$ projeto foi aprovado pelo comitê de ética médica do Hospital Universitário Professor Edgard Santos, UFBA, Salvador-Bahia.

Os indivíduos foram distribuídos aleatoriamente em dois grupos de 12, a depender do antígeno usado para o teste de Montenegro. No grupo I, utilizou-se antígeno solúvel de promastigotas de $L$. amazonensis preparado em nosso laboratório, e no grupo II, antígeno particulado de promastigotas de $L$. amazonensis produzido industrialmente (gentilmente cedido por Dr. Mauro Marzochi). Foram excluídos três indivíduos do grupo I que por ocasião da primeira reação de Montenegro apresentaram teste positivo.

Obtenção do antígeno de Leishmania. Para o teste intradérmico do grupo I e a avaliação da resposta imune in vitro, utilizou-se o antígeno solúvel de Leishmania preparado a partir de uma cepa previamente caracterizada por anticorpos monoclonais e isoenzimas como $L$. amazonensis ( MHOMBr 88 - Ba 125). Resumidamente, promastigotas em cultura foram lavadas com PBS pH 7,2 e rompidas pelo choque térmico e sonicação. Após centrifugação, o sobrenadante foi coletado e a concentração de proteínas medida pelo método de Lowry et $\mathrm{al}^{18}$. Para o teste intradérmico utilizou-se o antígeno na concentração de $250 \mu \mathrm{g}$ por $\mathrm{ml}$ em solução de PBS pH 7,2 com fenol a $0,4 \%$.
Considerando-se a importância do teste de Montenegro na prática clínica e na pesquisa, o objetivo do presente estudo foi avaliar comparativamente o poder imunizante de duas diferentes preparações de antígenos utilizados no teste de Montenegro, analisando a resposta imune in vivo e in vitro para antígenos de Leishmania, incluindo a determinação da produção de gama-interferon ( $y$-IFN), desde que esta citocina representa o principal fator estimulador de macrófagos para destruição de Leishmaniá.

Para o teste intradérmico no grupo II, foi utilizado antígeno total particulado de promastigotas da cepa $\mathrm{PH} 8$ de L. amazonensis (cepa de referência pela OMS) produzido por Biomanguinhos-FIOCRUZ, através de fracionamento subcelular por sonicação e choque térmico. $\mathrm{O}$ antígeno é preservado em solução de salina mertiolatada 1:10000, na concentração de $40 \mu \mathrm{g}$ de $\mathrm{N}$ protéico por $\mathrm{m}^{25}$, o equivalente a uma concentração de proteína de $250 \mu \mathrm{g}$ por $\mathrm{ml}$.

Reação de Montenegro. Foi administrado $0,1 \mathrm{ml}$ do antígeno de Leishmania a $3 \mathrm{~cm}$ da dobra cubital no antebraço dos indivíduos estudados, sendo a induração mensurada após 48 horas. A leitura foi realizada pela técnica de Sokal JE e cols ${ }^{39}$, e o teste considerado positivo quando a induração foi maior ou igual a $5 \mathrm{~mm}$. Os testes foram realizados em todos os indivíduos por ocasião da inclusão no estudo (dia 0), no dia 30, e repetido no dia 90 quando permanecia negativo.

Cultura de células mononucleares e produção de y-IFN. A produção de $y$-IFN foi avaliada no dia 0 , e 30 e 360 dias após o primeiro teste de Montenegro. As células mononucleares do sangue periférico (CMSP) foram obtidas através do gradiente de Ficoll-Hypaque (LMS; Organon Teknika corporation, Durham,NC). Resumidamente, CMSP $\left(3 \times 10^{6} / \mathrm{ml}\right)$ em meio de cultura RPMI completo foram estimuladas com antígenos de Leishmania $(10 \mu \mathrm{g} / \mathrm{ml})$ ou com o mitógeno PHA na diluição de 1:100. Após 72 horas de incubação a uma temperatura de $37^{\circ} \mathrm{C}$ e $5 \% \mathrm{CO}_{2}$, os sobrenadantes foram colhidos e os níveis de y-IFN determinados através da técnica de ELISA sanduíche. Os resultados foram expressos em $\mathrm{pg} / \mathrm{ml}$ baseados em uma curva padrão.

Análise estatística. As diferenças dos resultados dos testes de Montenegro entre os dias zero, 30 e 90, e as diferenças na proporção de indivíduos que produziram y-IFN nos dias 30 e 360 , comparado ao dia zero, foram analisadas através do teste exato de Fisher, enquanto que as diferenças entre os níveis de y-IFN dos dias zero com os dias 30 e 360 foram analisadas utilizando-se o teste Wilcoxon pareado.

\section{RESULTADOS}

O resultado dos testes de Montenegro, realizados no início do estudo, 30 e 90 dias após a primeira avaliação são mostrados na Tabela 1. Foi observado que no prazo de 30 dias, 3 dos 9 indivíduos (33\%) que utilizaram o antígeno solúvel (grupo I) e 5 dos 12 (42\%) que utilizaram o antígeno particulado (grupo II) converteram o teste para positivo. Uma terceira avaliação mostrou que após 90 dias da realização do teste intradérmico inicial, houve conversão do teste em mais 3 dos 9 indivíduos do grupo I e em mais 1 dos 12 do grupo II, ficando a positividade do teste em 67 e $50 \%$ para os grupos I e II, respectivamente. A diferença de 
Tabela 1 - Freqüência de positividade do Teste de Montenegro previamente, 30 e 90 dias após o primeiro teste intradérmico com antígeno de Leishmania.

\begin{tabular}{lccc}
\hline & Dia zero $(\%)$ & Dia $30(\%)$ & Dia $90(\%)$ \\
\hline Grupo I & 0 & 33 & $67^{*}$ \\
Grupo II & 0 & 42 & $50^{*}$ \\
\hline${ }^{*} p<0.05$ & & &
\end{tabular}

positividade do Montenegro entre os dias 0 e 90 foi estatisticamente significante tanto para o grupo I quanto para o grupo II $(\mathrm{p}<0,05)$.

As concentrações de y-IFN nos sobrenadantes de culturas estimuladas com antígeno de Leishmania são mostradas na Figura 1. No dia 30 após a realização do teste intradérmico, 7 (33\%) dos 21 indivíduos testados passaram a produzir y-IFN, sendo a média da produção nesses 7 indivíduos de $235 \pm 329 \mathrm{pg} / \mathrm{ml}$. Enquanto y-IFN não foi detectado em nenhum dos indivíduos na avaliação do dia zero, no dia 30 esta citocina foi detectada em 5 dos 9 indivíduos do grupo I $(p<0,05)$, com concentração média de $169 \pm 309 \mathrm{pg} / \mathrm{ml}$ (níveis variando entre 12 e $901 \mathrm{pg} / \mathrm{ml}$ ) e em dois dos 12 indivíduos do grupo II, com média de $11 \pm 36 \mathrm{pg} / \mathrm{ml}$ (níveis de 5 e 122pg/ml). Após 30 dias da realização do

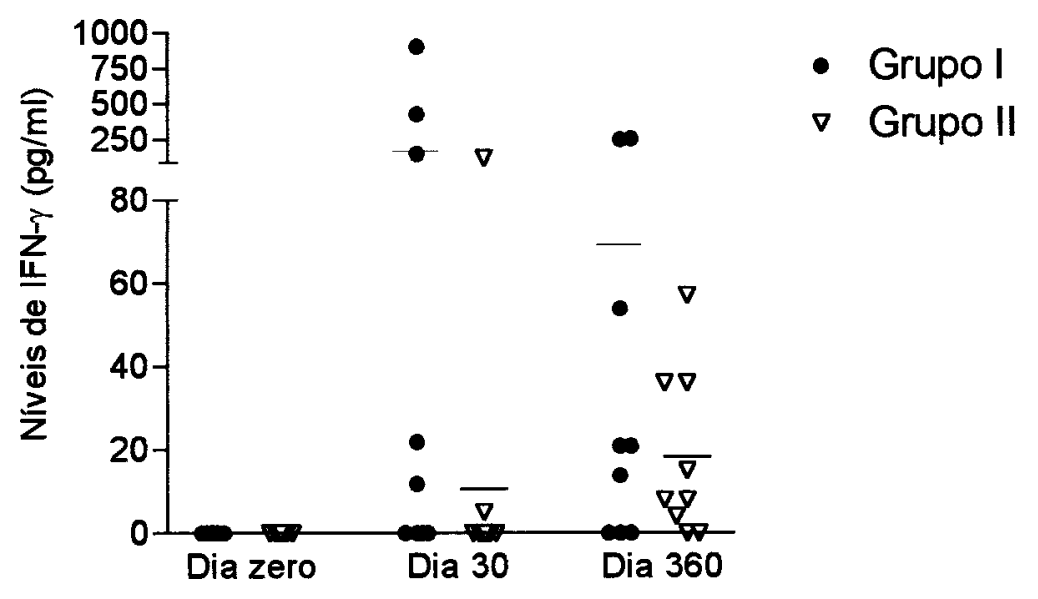

Figura 1 - Produção de g-IFN por células mononucleares do sangue periférico. IFN-y foi dosado previamente, 30 e 360 dias após o primeiro teste intradérmico em sobrenadantes de cultura estimuladas com antígeno de Leishmania utilizando-se a técnica ELISA. Cada símbolo representa um indivíduo.

primeiro teste intradérmico, 7 (78\%) de 9 indivíduos do grupo I e 6 (50\%) de 12 indivíduos do grupo II mostravam resposta imunológica para antígeno de Leishmania, definida por uma positividade do teste de Montenegro e/ ou produção de y-IFN em sobrenadantes de cultura.

A presença de y-IFN foi observada mesmo após 360 dias do primeiro teste em 13 de 18 indivíduos avaliados (72\%), sendo a média e desvio padrão da produção de y-IFN de $44 \pm 79 \mathrm{pg} / \mathrm{ml}$. Seis destes 13 indivíduos que produziram y-IFN no dia 360 já produziam esta citocina no dia 30 após o primeiro teste intradérmico. Na avaliação do dia 360, y-IFN foi detectado em seis dos nove indivíduos do grupo I que não produziram esta citocina na avaliação do dia zero $(p<0,05)$, com média de produção significativamente maior do a observada na avaliação inicial $(p<0,05)$ $(69 \pm 108 \mathrm{pg} / \mathrm{ml}$, níveis variando entre 14 e $258 \mathrm{pg} / \mathrm{ml})$. Enquanto a produção de y-IFN não foi também observada em indivíduos do grupo II na avaliação inicial, no dia 360 esta citocina foi detectada em sete indivíduos $(\mathrm{p}<0,05)$, com média de produção de $18 \pm 20 \mathrm{pg} / \mathrm{ml}$ (níveis variando entre 4 e $57 \mathrm{pg} / \mathrm{ml}$ ) (Figura 1). Em todos os indivíduos observou-se produção de y-IFN em culturas estimuladas com PHA (dado não mostrado).

\section{DISCUSSÃO}

O teste de hipersensibilidade tardia utilizando antígeno de Leishmania preconizado por Montenegro é um importante método para diagnóstico da leishmaniose tegumentar e é amplamente empregado em estudos epidemiológicos para a identificação de indivíduos expostos e sem doença e indivíduos curados de infecção causada pela Leishmania. O teste, entretanto, não é padronizado e vários preparados antigênicos em diferentes doses são descritos ${ }^{8}{ }^{32}$. Por esta razão, a avaliação do poder imunizante da reação de Montenegro, no presente estudo, foi realizada utilizando-se dois tipos de preparado antigênico correntemente empregados em estudos clínicos e epidemiológicos. Apesar das diferenças na preparação 
e nos conservantes utilizados, ambos os reagentes, quando aplicados na mesma dosagem, tiveram a capacidade de induzir resposta imune, in vitro e in vivo, ao antígeno de Leishmania.

O fenômeno de indução de hipersensibilidade tardia por repetição dos testes intradérmicos, em indivíduos sadios, é um assunto controverso na literatura. Enquanto Keystone et al $^{15}$ verificaram que cerca de $40 \%$ dos indivíduos avaliados apresentavam hipersensibilidade tardia após 6 semanas de exposição aos antígenos PPD, estreptoquinase, estreptodornase, Candida e da caxumba; Lesourd et $a{ }^{17}$ não observaram sensibilização do sistema imune nessas circunstâncias. Em relação ao antígeno de Leishmania parece ter relevância o intervalo de tempo entre a realização dos testes. Quando este intervalo foi de duas semanas, a positividade do $2^{\circ}$ teste ocorreu em mais de $60 \%$ dos indivíduos avaliados ${ }^{29}$, enquanto que não foi observado positividade do teste intradérmico quando o intervalo foi maior ou igual a um ano ${ }^{40}$.

Estudos prévios têm mostrado que células mononucleares de indivíduos sadios não expostos a Leishmania podem apresentar resposta linfoproliferativa e produção de y-IFN in vitro quando estimulados por antígenos desse parasita ${ }^{31914}$ 16. Este fenômeno pode ser explicado pela existência de reação cruzada, uma vez que epitopos presentes na Leishmania são documentadas em outros patógenos como Mycobacterium bovis e Trypanosoma cruzi ${ }^{38}$. Neste caso, poderia haver um número limitado de clones de células sensibilizadas que se expandiriam em contato com os antígenos de Leishmania. Embora não se possa afastar que reações cruzadas contribuam para explicar a conversão do teste do Montenegro após uma $2^{a}$ administração do antígeno, a observação neste estudo de que, na avaliação inicial não havia produção de y-IFN quando as células foram estimuladas, in vitro, com antígeno de Leishmania, aponta para a uma real capacidade destes antígenos serem imunogênicos. Deve-se ressaltar que os conservantes fenol e mertiolate poderiam interferir na leitura do teste intradérmico, mas a reação induzida por essas substâncias é, habitualmente, de hipersensibilidade imediata e este fenômeno também não explicaria a produção de citocinas in vitro.

O teste de Montenegro tem sido, ainda, aplicado como um marcador de resposta imune e da proteção contra a leishmaniose em indivíduos vacinados com antígeno bruto de Leishmania 522232428 . Como a proteção induzida por esta vacina ainda não foi comprovada e estudos com vacinas mostraram que a taxa de conversão do teste foi semelhante nos indivíduos vacinados e controles ${ }^{74}$, a utilização da reação de hipersensibilidade tardia como marcador da indução de resposta imune deve ser vista com cautela quando este teste é realizado previamente à administração da vacina. Recentemente, Marzochi et $\mathrm{al}^{21}$, encontraram uma taxa de conversão do teste de Montenegro de aproximadamente $66 \%$, tanto em indivíduos vacinados como no grupo controle. O conhecimento da produção de y-IFN induzida pelo teste de Montenegro também se reveste de grande importância, uma vez que a presença desta citocina vinha sendo relacionada com o poder imunizante da vacina em estudos onde a população era selecionada pela prévia realização do teste de Montenegro e, então, vacinadas para LTA. As observações do presente estudo apontam para a necessidade de uma melhor padronização das soluções de antígenos de Leishmania utilizadas para a realização desse teste intradérmico, e que devido a sua capacidade imunogênica ele não deve ser utilizado previamente ao uso de vacinas contra a LTA.

\section{AGRADECIMENTOS}

Os autores agradecem aos voluntários que participaram do estudo, a Virgínia Avelar pela preparação do antígeno de Leishmania, e a Dilma Simplício e Clenildo Bispo pelo auxílio técnico. Este estudo recebeu apoio financeiro do NIH AI 30639 e do Programa de Apoio aos Núcleos de Excelência (PRONEX).

\section{REFERÊNCIAS BIBLIOGRÁFICAS}

1. Abrahamson MA, Dietze R, Fruchi DM, Schwantz R, Kenney RT. Comparison of New and Old World leishmanins in an endemic region of Brazil. Clinical Infectious Disease 20:1292-1297, 1995.

2. Akuffo H, Darce M, Maasho K, Berhan, TY. In vivo evaluation of immune responses in leishmaniasis: the use of cross-species leishmanin preparations for skin testing. American Journal of Tropical Medicine and Hygiene 53:16-22, 1995.

3. Akuffo H, Maasho K, Howe R. Natural and acquired resistance to Leishmania: cellular activation of Leishmania aethiopica of mononuclear cells from unexposed individuals through the stimulation of natural killer (NK) cells. Clinical and Experimental Immunology 94:516-521, 1993.

4. Antunes CMF, Mayrink W, Magalhães PA, Costa CA, Melo MN, Dias M, Michalick MSM, Williams P, Lima AO, Vieira JBF, Schetini APM. Controlled field trials of a vaccine against New World cutaneous leishmaniasis. International Journal of Epidemiology 15:572-580, 1986.
5. Armijos RX, Weigel MM, Aviles H, Maldonado R, Racines J. Field trials of a vaccine against new world cutaneous leishmaniasis in an at-risk child population: safety, immunogenicity, and efficacy during the first 12 months of follow-up. Journal of Infectious Diseases 177:1352-1357, 1998.

6. Carvalho EM, Bacellar O, Reed S, Barral A, Rocha H. Visceral leishmaniasis: a disease associated with inability of lymphocytes to activate macrophages to kill Leishmania. Brazilian Journal of Medical and Biological Research 21:85-92, 1988.

7. Castes M, Blackwell J, Trujillo D, Formica S, Cabrera M, Zorilla G, Rodas A, Castellanos PL, Convit J. Immune response in healthy volunteers vaccinated with killed leishmanial promastigotes plus BCG. I: Skin test reactivity, T-cell proliferation and IFN-gamma production. Vaccine 12:1841-1851, 1994.

8. Cuba-Cuba CA, Marsden PD, Barretto AC, Jones TC, Richards F. The use of different concentrations of leishmanial antigen in skin 
testing to evaluate delayed-hypersensitivity in American cutaneous leishmaniasis. Revista da Sociedade Brasileira de Medicina Tropical 18:231-236, 1985.

9. Follador I, Araujo C, Cardoso MA, Tavares-Neto J, Baral A, Miranda JC, Bitencourt A, Carvalho EM. Outbreak of American cutaneous leishmaniasis in Canoa, Santo Amaro, Bahia, Brazil. Revista da Sociedade Brasileira de Medicina Tropical 32: 497503, 1999.

10. Furtado T. Critérios para diagnóstico da leishmaniose tegumentar americana. Anais Brasileiros de Dermatologia 65:51-86, 1980.

11. Guedes ACM, Cucé LC, Furtado, T. Avaliação imunológica e histopatólogica de reação de Montenegro. Anais Brasileiros de Dermatologia 65:34S-405, 1990.

12. Guerra MOP, Furtado T, Barros GC, Sessa PA, Daher VR. Infecção subcliníca na leishmaniose tegumentar americana. Anais Brasileiros de Dermatologia 60:365-369, 1985.

13. Isaza MR. La reacion de Montenegro en la epidemiologia de la Ieishmaniasis sudamericana. Boletin Oficina Sanitaria Panamericana 89:130-136, 1980.

14. Kemp M, Hansen MB, Theander TG. Recognition of Leishmania antigens by $T$ lymphocytes from nonexposed individuals. Infection and Immunity 60:2246-2251, 1992.

15. Keystone EC, Demerieux P, Gladman D, Poplonski L, Piper S, Buchanan R. Enhanced delayed-hypersensitivity skin test reactivity with serial skin testing in healthy volunteers. Clinical Experimental Immunology 40:202-205,1980.

16. Kurtzhals JA, Kemp M, Poulsen LK, Hansen MB, Kharazmi A, Theander TG. Interleukin-4 and interferon-gamma production by Leishmania stimulated peripheral blood cells from non-exposed individuals. Scandinavian Journal of Immunology 41:343-349, 1995.

17. Lesourd BM, Wang A, Moulias R. Serial delayed cutaneous hypersensitivity skin testing with multiple recall antigens in healthy volunteers: booster effect study. Annals of Allergy 55:729-735, 1985.

18. Lowry OH, Rosenbrough NJ, Farr AL, Randal RJ. Protein measurement with the folin phenol reagent. Journal of Biological Chemistry 193:245, 1951.

19. Maasho K, Akuffo $\mathrm{H}$. Recognition of Leishmania antigens by $\mathrm{T}$ cells of non-exposed individuals produce cytokines fractions of Leishmania promastigotes. Scandinavian Journal Immunology 11:179-184, 1992.

20. Martins-Netto E. Avaliação de procedimentos imunodiagnósticos numa área endêmica de leishmaniose tegumentar na Bahia. Dissertação de Mestrado. Universidade Federal da Bahia. Salvador, 1990.

21. Marzochi KB, Marzochi MA, Silva AF, Grativol N, Duarte R, Confort EM, Modabber F. Phase I of an inactivated vaccine against American tegumentary leishmaniasis in normal volunteers in Brazil. Memórias do Instituto Oswaldo Cruz 93:205-212, 1998.

22. Mayrink W, Da Costa CA, Magalhães PA, Melo MN, Dias M, A Oliveira Lima, Michalick MSM, Williams, P. A field trial of a vaccine against American dermal leishmaniasis. Transactions of the Royal Society of Tropical Medicine and Hygiene 73:385-387, 1979.

23. Mayrink W, Magalhães $M$, Dias $M$, Da Costa CA, Melo MN, Oliveira Lima A. Response to Montenegro antigen after immunization with killed Leishmania promastigotes. Transactions of the Royal Society of Tropical Medicine and Hygiene 72:676, 1978.

24. Mayrink W, Williams P, Da Costa CA, Magalhães PA, Melo MN, Dias M, Lima AO, Michalick MSM, Carvalho FF, Barros G, Sessa $\mathrm{PA}$, De Alencar JE. An experimental vaccine against American dermal leishmaniasis: experience in the State of Espírito Santo, Brazil. Annals of Tropical Medicine Parasitology 79:259-269, 1985.

25. Melo MN, Mayrink W, Costa CA, Magalhães PA, Dias M, Williams P, Araújo FG, Coelho MV, Batista SM. Padronização do antígeno de Montenegro. Revista do Instituto de Medicina Tropical de São Paulo 19:161-164, 1977.

26. Mendonça SC, De Luca P, Mayrink W, Restom T, ConceiçãoSilva F, Da Cruz AM, Bertho A, Da Costa C, Genaro O, Toledo V. Characterization of human T-lymphocyte mediated immune response induced by a vaccine against American tegumentary leishmaniasis. American Journal of Tropical Medicine and Hygiene 53:195-201, 1995.

27. Montenegro J. Cutaneous reaction in leishmaniasis. Archives of Dermatology and Syphilis 13:187-184, 1926.

28. Nascimento E, Mayrink W, Da Costa CA, Michalick MSM, Melo MN, Barros GC, Dias M, Antunes CMF, Lima MS, Taboada DC, Liu TY. Vaccination of humans against cutaneous leishmaniasis: Cellular and humoral immune response. Infection and Immunity 58:2198-2203, 1990.

29. Nascimento MDSB, Alcântara-Neves NM, Muniz MEB, Nunes SF, Paranhos M, Carvalho LCP. Induction and modulation of the immune response to Leishmania by Montenegro's skin test. Transactions of the Royal Society of Tropical Medicine and Hygiene 87:91-93, 1993.

30. Pellegrino V, Furtado T. A reação intradérmica no diagnóstico da leishmaniose tegumentar americana. Observações com antígenos solúveis de Leishmania braziliensis. Medicina Cutanea Ibero Latino Americana 15:105-116, 1960.

31. Pessoa SB, Pestana BR. A intradermorreação de Montenegro nas campanhas sanitárias contra a leishmaniose. Arquivos de Higiene, São Paulo 6:124-137, 1941.

32. Rotberg A. Contribuição para o estudo da alergia na leishmaniose tegumentar americana. Tese, Universidade de São Paulo, 1951.

33. Sessa PA, Falqueto A, Barros GCB, Varejão JBM. Resultados da reação de Montenegro em pacientes com leishmaniose tegumentar americana, autócnes do Estado do Espírito Santo. Revista da Associação Médica Brasileira 37:115-118, 1991.

34. Sharples CE, Shaw MA, Castes M, Convit J, Blackwell JM. Immune response in healthy volunteers vaccinated with BCG plus killed leishmanial promastigotes: antibody responses to mycobacterial and leishmanial antigens. Vaccine 12:1402-1412, 1994.

35. Shaw JJ, Lainson R. Leishmaniasis in Brazil: X. Some observations on intradermal reactions to different trypanosomatid antigens of patients suffering from cutaneous and mucocutaneous leishmaniasis. Transactions of the Royal Society of Tropical Medicine and Hygiene 69:323-335, 1975.

36. Silveira TG, Teodoro U, Lonardoni UM, Guilherme AL, Toledo MJ. Epidemiologic aspects of cutaneous leishmaniasis in an endemic area of the state of Paraná, Brazil. Cadernos de Saúde Pública 12:141-147, 1996. 
37. Silveira FT, Lainson R, Shaw IL, De Souza AA, Ishikaea PA, Braoa RR, Cutaneous Leishmaniasis due to Leismmania (Leishmania) amazonensis in Amazonian Brazil, and the significance of a negative Montenegro skin-test in human infections. Transactions of the Royal Society of Tropical Medicine and Hygiene 35:735-738, 1991.

38. Smrkovski LL, Larson CL. Antigenic cross-reactivity between Mycobacterium bovis (BCG) and Leishmania donovani. Infection and Immunity 18:561-562, 1977.
39. Sokal JE. Measurement of delayed skin test responses. New England Journal of Medicine 293:501-502, 1975.

40. Weigle KA, Valderrama L, Arias AL, Santrich C, Saravia NG. Leishmanin skin test stantardization and evaluation of safety, dose, storage, longevity of reaction and sensitization. American Journal of Tropical Medicine and Hygiene 44:260-271, 1991.

41. World Health Organization. Leishmaniasis. Geographical distribution, May, 2000. 